\title{
Review of: "A unified drug-target interaction prediction framework based on knowledge graph and recommendation system"
}

shuyu wang

Potential competing interests: The author(s) declared that no potential competing interests exist.

KGE_NFM has shown successful DTI prediction by leveraging KG and recommendation system. It efficiently utilizes the low-dimensional characterization from KGE, which is very impressive. Moreover, it enables the prediction more robust by integrating multi-modal data.

However, this paper failed to compare some state-of-the-art works on DTI tasks, such as durgVQA, MONN, GanDTI, and etc. If the KGE_NFM can also outperform these works on the same tasks, it could better demonstrate the works' efficacy. 